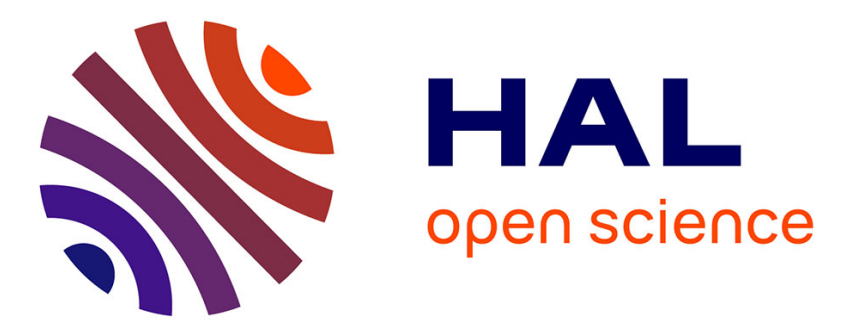

\title{
B Formal Validation of ERTMS/ETCS Railway Operating Rules
}

Rahma Ben Ayed, Simon Collart-Dutilleul, Philippe Bon, Akram Idani, Yves Ledru

\section{- To cite this version:}

Rahma Ben Ayed, Simon Collart-Dutilleul, Philippe Bon, Akram Idani, Yves Ledru. B Formal Validation of ERTMS/ETCS Railway Operating Rules. 4th International ABZ Conference, Jun 2014, France. p124-129. hal-01066368

\section{HAL Id: hal-01066368 https://hal.science/hal-01066368}

Submitted on 19 Sep 2014

HAL is a multi-disciplinary open access archive for the deposit and dissemination of scientific research documents, whether they are published or not. The documents may come from teaching and research institutions in France or abroad, or from public or private research centers.
L'archive ouverte pluridisciplinaire HAL, est destinée au dépôt et à la diffusion de documents scientifiques de niveau recherche, publiés ou non, émanant des établissements d'enseignement et de recherche français ou étrangers, des laboratoires publics ou privés. 


\title{
$B$ Formal Validation of ERTMS/ETCS Railway Operating Rules
}

\author{
Rahma Ben Ayed ${ }^{1}$, Simon Collart-Dutilleul ${ }^{1}$, Philippe Bon ${ }^{1}$, \\ Akram Idani $^{2}$, and Yves Ledru ${ }^{2}$ \\ 1 Univ. Nord de France, IFSTTAR/COSYS-ESTAS, 20 rue Elisée Reclus, F-59650, \\ Villeneuve d'Ascq, France \\ \{rahma.ben-ayed, simon.collart-dutilleul, philippe.bon\}@ifsttar.fr \\ http://www.ifsttar.fr \\ 2 UJF-Grenoble 1/Grenoble-INP/UPMF-Grenoble 2/CNRS, LIG UMR 5217, \\ F-38041, Grenoble, France \\ \{akram.idani, yves.ledru\}@imag.fr \\ http://www.liglab.fr
}

\begin{abstract}
The $B$ method is a formal specification method and a means of formal verification and validation of safety-critical systems such as railway systems. In this short paper, we use the B4MSecure tool to transform the UML models, fulfilling requirements of European Railway Traffic Management System (ERTMS) operating rules, into $B$ specifications in order to formally validate them.
\end{abstract}

Keywords: Railway operating rules, UML models, Role Based Access Control, $B$ method, formal validation.

\section{Introduction}

ERTMS [6] is the European Railway Traffic Management System which is designed to replace the national on-board railway systems in Europe in order to make rail transport safer and more competitive, and to improve cross-border connections. ERTMS includes the European Train Control System (ETCS) which specifies the on-board equipment and its communication with the trackside.

The aim of our work is to confront the European specifications with the national operating rules, as well as the use of formal models to validate whether a given scenario fulfills the specification regarding the functional and safety requirements. We propose to model a nominal scenario of Movement Authority $(M A)$, extracted from ERTMS operating rules, and to translate it into $B$ specifications in order to validate it.

In the following section, an overview of the nominal scenario $M A$ is given and its UML models are described. Section 3 highlights the $B$ formal validation after an automatic translation of these models into $B$ specifications using B4MSecure. Finally, section 4 concludes this paper. 


\section{Movement Authority Overview}

Movement Authority ( $M A$ ) is an authorization given to a train to move to a given point as a supervised movement. Some features can be used to define an $M A$, such as sections subdividing it, the time-out value attached to each section, etc. The $M A$ function unfolds with interactions between the OnboardSafetyManagement (the on-board computer-based machine), the TracksideSafetyManagement (the trackside computer-based machine), and the Driver, as follows:

MA.1 The OnboardSafetyManagement requests an MA to the TracksideSystem.

MA.2 The TracksideSafetyManagement $\underline{\text { receives }}$ the $M A$ request from the TracksideSystem.

MA.3 The TracksideSafetyManagement proposes an MA to the TracksideSystem after creating it. It can also modify and/or delete the $M A$.

MA.4 The OnboardSafetyManagement receives the proposed $M A$ from the TracksideSystem, authorizes it and processes the $M A$ authorization in order to be displayed in the Driver Machine Interface (DMI).

MA.5 The Driver reads the authorized $M A$.

Each step of this scenario represents a permission to do an action on an entity by a role. On this basis, 3 roles (OnboardSafetyManagement, TracksideSafetyManagement, Driver), 3 system entities (TracksideSystem, MA, DMI) and 10 possible permissions (underlined actions) can be extracted.

Our approach consists in, on the one hand, the modeling of ERTMS operating rules in semi-formal UML notations with their graphical views and dedicated profiles extensions taking into account various aspects (structural, dynamic, behavioural, etc.), and on the other hand, their validation and verification with a formal $B$ method with its mathematical notations and automated proof. The combination of these two notations has been studied and several approaches of UML to $B$ translation have been proposed, cited in 3. In order to model the scenario above, we use B4MSecure platform supporting the UML/B modeling process and lying within the scope of Model Driven Engineering (MDE).

For the sake of concision, the B4MSecure platform [7] is briefly presented. As an Eclipse platform, it is dedicated to formally validate a functional UML model enhanced by an access control policy. It uses a Role Based Acces Control (RBAC) profile inspired from SecureUML profile [4. This profile aims at specifying information related to access control in order to model roles and their permisssions. This platform acts in 3 steps: a functional UML class diagram specifying system entities, security UML models with an access control policy and the translation of both models into $B$ specifications.

Following the three-stepped approach of B4MSecure, a functional UML class diagram containing all system entities as classes and the relationships between them is built. Then, security UML class diagrams enhance the functional model by expressing which role has the permission to perform a given action in the railway system: a class diagram dedicated to the roles and others dedicated to 
the access control policies which are based on permissions linking the roles to the entities, such as the access control of the $M A$ in Fig. 1. A permission is modeled as a UML association class, between a role and a class of the functional model, with a stereotype Permission. For instance, MA.4 is modeled in Fig. 1 by the permission of the OnboardSafetyManagement to authorize the $M A$. All these diagrams are translated to $B$ specifications.

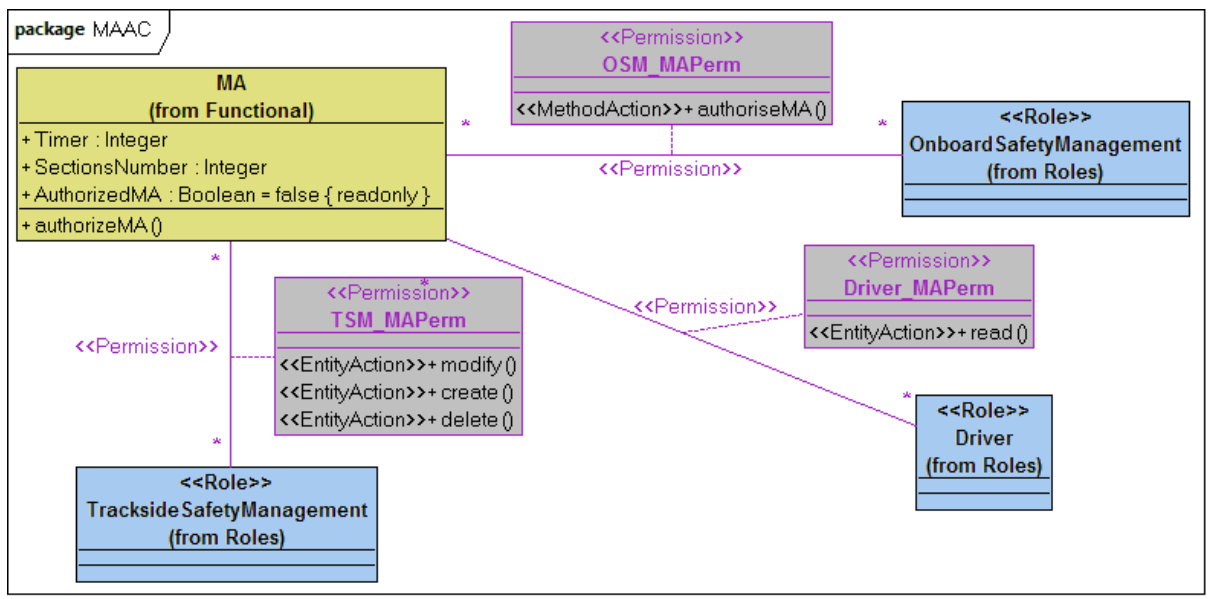

Fig. 1. Roles and permissions associated with MA

\section{$3 \quad B$ Formal Validation of Movement Authority}

The functional model is translated into a unique $B$ machine, named Functional, and permissions are translated into a $B$ machine, named $R B A C \_$Model. As shown in Fig. 2. the functional formal model follows a classical translation scheme similar to [5]. The RBAC_Model adds variables about permissions and roles. For example, PermissionAssignement is a total function from PERMISSIONS to the cartesian product (ROLES ${ }^{*}$ ENTITIES), and isPermitted is a relation between ROLES and Operations sets. PERMISSIONS, ENTITIES and Operations are the sets defined in $R B A C_{-}$Model, while $R O L E S$ is a set defined in the included UserAssignments machine. Initialization of these variables is conformant to the SecureUML model. Then, initialization proof obligation, produced by the AtelierB prover for these variables, allows to verify whether the SecureUML model respects RBAC well-formedness rules such as no cycles in role hierarchy, etc. The operations of the security formal model encapsulate the operational part of the functional formal model. Each functional operation is associated with an operation in the security model verifying that a user has permission to call the functional operation. For instance, secure_MA_-authorizeMA operation of $R B A C_{-}$Model checks the permissions associated with the functional operation $M A \_$authorizeMA. Secured operations add a statement in the postcondition 


\begin{tabular}{|l} 
Machine \\
Functional \\
SETS \\
MA_AS; $\ldots$ \\
ABSTRACT_VARIABLES \\
MA, $\ldots$ \\
INVARIANT \\
MA <: MA_AS \& $\ldots$ \\
INITIALISATION \\
MA := \{\} II ... \\
OPERATIONS \\
MA__authorizeMA (Instance) $=$ \\
PRE \\
Instance : MA \& \\
MA__AuthorizedMA (Instance) $=$ FALSE \\
THEN \\
MA__AuthorizedMA (Instance) $:=$ TRUE \\
END; $\ldots$ \\
END
\end{tabular}

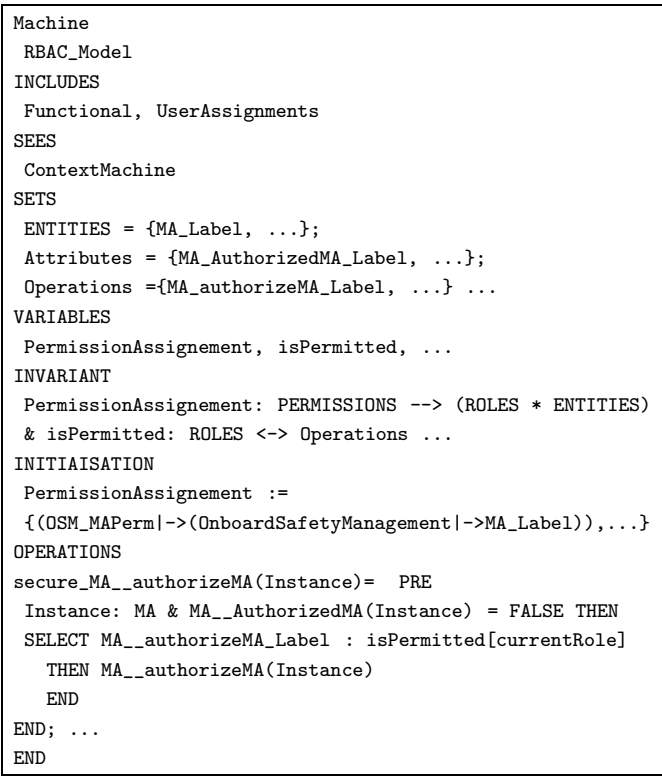

Fig. 2. Functional and $R B A C_{-}$Model machines

e.g SELECT MA_-authorizeMA_Label: isPermitted[currentRole] in order to verify whether MA__authorizeMA_Label is allowed to the connected user using a particular role. Indeed, isPermitted computes, from the initial state, the set of authorized functional operations for each role.

UML models of extracted ERTMS/ETCS operating rules containing 7 functional classes, 5 roles and 17 permissions are transformed into 830 lines of functional formal model and 1545 lines of security formal model. We use the ProB animator in order to validate these specifications. A first animation checks the nominal behaviour of Movement Authority. Then variants of this animation check that the given permissions forbid the execution of secure actions by unauthorized roles, since a secure action can be performed only with a permission given to a role. The ability of the system specified by the class diagram to play the ERTMS scenarios is checked through animations of the corresponding transformed B model. These animations validate the permissions assigned to each role. But they don't check that the sequence of actions models the MA protocol. Actually, the sequence of actions is defined in the animation by the user, but it is not embedded in the UML/B model. This can be resolved by adding some contraints as preconditions in secured operations. Nevertheless, adding these conditions breaks the consistency between the UML model and the B machine. Owing to the lack of dynamic aspects in UML class diagrams, we intend to explore more UML diagrams as future work. Then we will focus on enriching UML class diagrams with, for instance, sequence diagrams which model the ordered 
interactions in scenarios and deriving $B$ specifications from them in order to validate system's behavior.

At this stage, safety requirements have not yet been integrated to $B$ specifications. As further work, we will consider enriching the $B$ specifications with safety properties stemming from safety requirements of the ERTMS operating rules in order to formally verify them using the $B$ prover. Moreover, SysML requirement diagrams combined with our UML diagrams may guarantee the traceability aspects of system requirements when they will be translated to $B$ specifications.

\section{Conclusion}

In this short paper, we have presented a Movement Authority function extracted from the ERTMS/ETCS operating rules. This function was modeled using UML graphical notations and then translated automatically, via the B4MSecure platform, into $B$ specifications which were checked successfully using the ProB animator. The combination of UML/B aims to ease the understanding of the system with the graphical notations of UML and formally validate system requirements with $B$ formal notations. Research works done in the Selkis project [1, 2] and 3 show the efficiency of this platform and its different steps leading to the formal validation of scenarios in the healthcare Information Systems by seeking for malicious sequences of operations. However, in this paper, we show the use of this existant platform in another context related to distributed railway systems and their operating rules.

Acknowledgements This research is funded by the PERFECT project (ANR12-VPTT-0010) and is partly supported by the SELKIS project (ANR-08-SEGI018).

\section{References}

1. Ledru, Y., Idani, A., Milhau, J., Qamar, N., Laleau, R., Richier, J.-L., Labiadh, M.-A.: Taking into Account Functional Models in the Validation of IS Security Policies. In: Salinesi, C., Pastor, O. (eds.) CAiSE 2011 Workshops. LNBIP, vol. 83, pp. 592-606. Springer, Heidelberg (2011)

2. Milhau, J., Idani, A., Laleau, R., Labiadh, M.A., Ledru, Y., Frappier, M.: Combining UML, ASTD and B for the formal specification of an access control filter. In: Innovations in Systems and Software Engineering, vol. 7, pp. 303-313. Springer (2011)

3. Idani, A., Labiadh, M.A., Ledru, Y.: Infrastructure dirigée par les modèles pour une intégration adaptable et évolutive de UML et B. Ingénierie des Systèmes d'Information Journal 15, 87-112 (2010)

4. Lodderstedt, T., Basin, D., Doser, J.: SecureUML: A UML-Based Modeling Language for Model-Driven Security. In: Jézéquel, J.-M., Hussmann, H., Cook, S. (eds.) UML 2002. LNCS, vol. 2460, pp. 426-441. Springer, Heidelberg (2002) 
5. Laleau, R., Mammar, A.: An overview of a method and its support tool for generating B specifications from UML notations. In: Proceedings of the 15th IEEE Interational Conference on Automated Software Engieering, ASE 2000, pp. 269-272. IEEE Computer Society, Washington (2000)

6. ERTMS, http://www.ertms.net

7. B4MSecure, http://b4msecure.forge.imag.fr 